\title{
HRJ
}

V.3 n.14 (2022)

Recebido: 30/11/2021

Aceito: 09/12/2021

\section{Segurança do paciente na antibioticoprofilaxia cirúrgica: revisão integrativa}

\author{
Marcely Feitosa do Carmo ${ }^{1}$ \\ Lauane Rocha Itacarambi ${ }^{2}$ \\ Aparecida Keilly Nogueira Silva ${ }^{3}$ \\ Marcelo Moreira Corgozinho ${ }^{4}$ \\ Jacqueline Ramos de Andrade Antunes Gomes ${ }^{5}$ \\ Verônica Santos de Melo ${ }^{6}$ \\ Ruth Silva Matos ${ }^{7}$ \\ Gleyce Mikaelle da Costa Quirino ${ }^{8}$ \\ Roseli Fátima Rosa dos Santos 9 \\ Gilberto Sabino da Silva ${ }^{10}$ \\ Cinthya Marques do Nascimento ${ }^{11}$
}

\footnotetext{
${ }^{1}$ Enfermeira Residente do Programa de Residência de Enfermagem em Centro Cirúrgico ESCS/FEPECS/SES/DF

${ }^{2}$ Enfermeira Residente do Programa de Residência de Enfermagem em Centro Cirúrgico ESCS/FEPECS/SES/DF

${ }^{3}$ Enfermeira Preceptora do Programa de Residência de Enfermagem em Centro Cirúrgico ESCS/FEPECS/SES/DF

${ }^{4}$ Enfermeiro Tutor do Programa de Residência de Enfermagem em Centro Cirúrgico ESCS/FEPECS/SES/DF

${ }^{5}$ Enfermeira Doutora em Ciências da Saúde - UnB, Coordenadora do Programa de Residência de Enfermagem em Centro Cirúrgico ESCS/FEPECS/SES/DF

${ }^{6}$ Enfermeira Residente do Programa de Residência de Enfermagem em Centro Cirúrgico ESCS/FEPECS/SES/DF

${ }^{7}$ Enfermeira Residente do Programa de Residência de Enfermagem em Centro Cirúrgico ESCS/FEPECS/SES/DF

${ }^{8}$ Enfermeira Residente do Programa de Residência de Enfermagem em Centro Cirúrgico ESCS/FEPECS/SES/DF

${ }^{9}$ Enfermeira Preceptora do Programa de Residência de Enfermagem em Centro Cirúrgico ESCS/FEPECS/SES/DF

${ }^{10}$ Enfermeiro Preceptor do Programa de Residência de Enfermagem em Centro Cirúrgico ESCS/FEPECS/SES/DF

${ }^{11}$ Enfermeira UTI/HUB e Colaboradora do Programa de Residência de Enfermagem em Centro Cirúrgico ESCS/FEPECS/SES/DF
} 


\title{
RESUMO
}

Introdução: É estimado pela OMS (Organização Mundial de Saúde) que milhares de pessoas sofrem de algum dano causado pela insegurança que é prestada pelo serviço de saúde, seja ele privado ou público. Esses danos podem ser reversíveis ou irreversíveis podendo chegar ao óbito. Objetivo: Descrever, a partir da literatura, os fatores predisponentes para falhas na antibioticoprofilaxia cirúrgica. Metodologia: Trata-se de uma revisão integrativa da literatura, que aborda a temática da antibioticoprofilaxia cirúrgica. As etapas foram analisadas com utilização das recomendações do Prisma (Preferred Reporting Items for Systematic Reviews and Meta-Analyses). Resultados: Com análise de 57 estudos, considerando 8 que abordavam a temática sobre segurança do paciente, antibioticoprofilaxia, e centro cirúrgico, não houve duplicidade nas bases e foram excluídos os artigos que tratavam da antibioticoprofilaxia em especialidades. Conclusão: Os cuidados relacionados à segurança do paciente na antibioticoprofilaxia nas cirurgias, estão diretamente relacionados ao cumprimento das diretrizes publicado pela OMS, e estes implicam na redução dos casos relacionados a infecção de sítio cirúrgico, tal como diminuição das taxas de complicações e mortalidade.

Palavras chaves: Antibioticoprofilaxia. Segurança do paciente; Centro Cirúrgico.

\section{Patient safety in surgical antibioticprophylaxis: integrative review}

\begin{abstract}
Introduction: the WHO (World Health Organization) that thousands of people suffer from some damage caused by the insecurity that is provided by the health service, whether private or public, estimates it. These damages can be reversible or irreversible and can lead to death. Objective: To describe, based on the literature, the predisposing factors for failures in surgical antibiotic prophylaxis. Methodology: This is an integrative literature review that addresses the topic of surgical antibiotic prophylaxis. The steps were analyzed using the Prisma recommendations (Preferred Reporting Items for Systematic Reviews and MetaAnalyses). Results: With an analysis of 57 studies, considering 8 that addressed the issue of patient safety, antibiotic prophylaxis, and operating room, there was no duplication in the databases and articles dealing with antibiotic prophylaxis in specialties were excluded. Conclusion: The care related to patient safety in antibiotic prophylaxis in surgeries is directly related to compliance with the guidelines published by the WHO, and these imply a reduction in cases related to surgical site infection, as well as a reduction in the rates of complications and mortality.
\end{abstract}

Keywords: Antibiotic prophylaxis. Patient safety; Surgery Center.

\section{INTRODUÇÃO}

É estimado pela OMS (Organização Mundial de Saúde) que milhares de pessoas sofrem de algum dano causado pela insegurança que é prestada pelo serviço de saúde, seja ele privado ou público. Esses danos podem ser reversíveis ou irreversíveis podendo chegar ao óbito. A Segurança do Paciente tem como objetivo reduzir ao mínimo os danos e riscos que o paciente pode estar exposto, proporcionando uma segurança no serviço prestado. Os 
incidentes que resultam em algum dano ao paciente são chamados de eventos adversos, sendo estes na maioria das vezes evitáveis, onde houve uma falhar no cuidar, no garantir uma segurança no atendimento ${ }^{1}$.

As ISC (Infecção de Sítio Cirúrgico) implicam diretamente no aumento de permanência hospitalar, quando se comparadas com aqueles pacientes que não foram acometidos por infecção, sendo que a alta hospitalar ocorre em até dois dias. Já os pacientes acometidos por ISC tem sua permanência aumentada, fato que gera o aumento do gasto hospitalar com oneração ao sistema de saúde pública. Além disso, outros impactos na vida desse paciente estão associados a uma maior resistência bacteriana devido ao uso prolongado de antibiótico, o que leva à necessidade do uso de antibióticos mais resistentes. Com maior gravidade a esse paciente é saber que este tipo de infecção pode levar a óbito. Alguns dados epidemiológicos trazem uma porcentagem de entre 20 e $30 \%$ de ISC, o que leva esse paciente a uma nova hospitalização ${ }^{2}$.

A busca por intervenções cirúrgicas tem crescido de forma considerável, aproximadamente são realizadas entre 187 a 281 milhões de cirurgia anualmente. Nos Estados Unidos, o quantitativo referente as cirurgias registram 16 milhões de intervenções cirúrgicas. Os custos relacionados a ISC, decorrente de uma intervenção cirúrgica podem chegar a US\$1,6 bilhão anuais ${ }^{3}$.

As cirurgias são classificadas de acordo com seu grau de contaminação microbiana, sendo definidas da seguinte forma: a) cirurgias limpas são as que não existem inflamação no local operado, sem quebra de técnica asséptica e penetração do trato respiratório, gastrintestinal ou geniturinário; b) cirurgias potencialmente contaminadas são aquelas em que há inserção sob condições controladas dos tratos respiratório, gastrintestinal ou geniturinário; c) cirurgias contaminadas incluem as feridas traumáticas, abertas e recentes, com presença de 
inflamação não purulenta; e d) cirurgias infectadas são as que nos tecidos existem presença de supuração local, necrose, e feridas traumáticas sujas ${ }^{1}$.

Diante dessas classificações das cirurgias é complexo dizer qual a dose e o antibiótico ideal para cada paciente, e, assim, existem protocolos e guidelines que tratam do assunto para fundamentar a escolha de acordo com a microbiota prevalente. Estudos publicados recentemente pela Infectious Diseases Society of America (IDSA) e a Society for Healthcare Epidemiology of America (SHEA) declararam consenso a respeito da administração de antibióticos, que devem ser intervenções projetadas para melhorar e medir o uso apropriado de antibióticos, incluindo dosagem, duração da terapia e via de administração - o uso adequado de antibiótico promove benefícios como a diminuição de eventos adversos ${ }^{4}$.

Houve uma preocupação em relação a temática segurança do paciente a partir da publicação do relatório To Erris Human: Building a Safer Health Care System, no ano de 1999, quando houve relatos dos dados alarmantes referentes aos erros envolvendo o processo do cuidado em saúde, e os danos que podem atingir aos pacientes, se tornando essa temática pauta prioritária em muitos países pelo mundo. Para que realmente exista uma segurança do paciente nas instituições, é preciso haver uma estruturação nas comunicações, no aprendizado da organização, comprometimento da equipe, voltada a dar valor a temática, sem buscar apenas a abordagem que pune o erro ${ }^{5}$.

Nesse sentido, a segurança do paciente é uma temática de grande preocupação, que fez com que a OMS juntamente com seus Estados membros firmasse uma aliança, a Aliança Mundial para Segurança do Paciente lançada em 2004, com o intuito de chamar a atenção dos profissionais de saúde para um maior comprometimento visando uma melhora na assistência à saúde tão como o desenvolvimento de políticas públicas para boas práticas assistenciais. A cada ano é lançado um novo desafio, e no segundo ano o desafio foi focado em segurança cirúrgica, com objetivo de elevar os padrões de qualidade nos serviços de saúde em todo o 
mundo, dentre eles pode-se destacar prevenção de infecção de sitio cirúrgico. Com base nesse cenário é observado o dever por buscas de estudos que contemplem o assunto ${ }^{1}$.

Portanto, objetiva-se através dessa pesquisa descrever a partir da literatura o perfil temático das publicações que versam sobre segurança do paciente na antibioticoprofilaxia cirúrgica.

\section{METODOLOGIA}

No que tange ao desenho do estudo, trata-se de uma revisão integrativa da literatura, que aborda a temática da antibioticoprofixia cirúrgica. As etapas desta pesquisa foram analisadas com a utilização das recomendações do Preferred Reporting Items for Systematic Reviews and Meta-Analyses (Prisma) ${ }^{6}$.

A questão norteadora para seleção dos textos foi estruturada a partir da estratégia focada no participante, área de interesse, comparação e Outcomes/Resultados (Pico), a saber: qual o perfil temático das publicações que versam sobre segurança do paciente na antibioticoprofilaxia cirúrgica? Assim, os parâmetros definidos na estratégia de busca foram: população (paciente cirúrgico), interesse do estudo (antibioticoprofilaxia), contexto (sem comparação) e desfecho (segurança do paciente) $)^{6}$.

\section{Estratégia de busca bibliográfica}

A busca bibliográfica foi realizada no Portal da Biblioteca Virtual de Saúde (BVS) entre junho e julho de 2020, na base de dados: Medical Literature Analysis and Retrieval System Online (Medline) e Literatura Latino-americana e do Caribe em Ciências da Saúde (Lilacs).

Os descritores selecionados na busca foram os termos do MESH (Medical Subject Headings), também contemplados nos Descritores em Ciências da Saúde (DeCS), a saber: 
"antibioticoprofilaxia" e "segurança do paciente" e "antibiotic prophylaxis" e "patient safety". Nas bases de dados Medline e Lilacs, identificando inicialmente 52 textos na primeira ("antibiotic prophylaxis"[MeSH Terms] OR ("antibiotic"[All Fields] AND "prophylaxis"[All Fields]) OR "antibiotic prophylaxis"[All Fields]) AND ("patient safety"[MeSH Terms] OR ("patient"[All Fields] AND "safety"[All Fields]) OR "patient safety"[All Fields]); e apenas cinco (5) textos na segunda base ("antibioticoprofilaxia" AND "segurança do paciente").

Imagem 1. Processo de seleção dos artigos

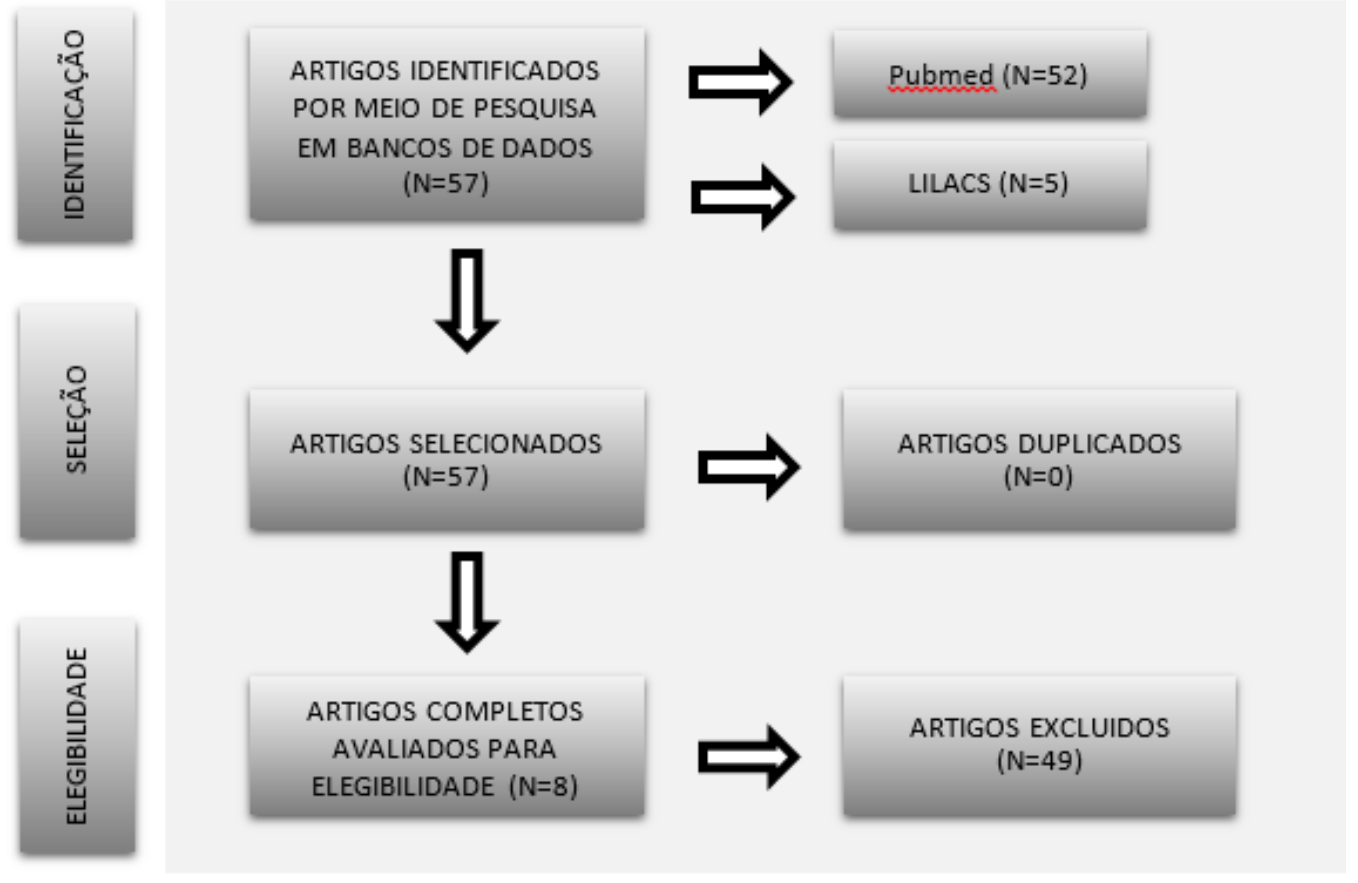

Fonte: Elaborado pela autora

\section{Critérios de elegibilidade}

Para a aquisição dos dados necessários na construção da presente pesquisa foram incluídos artigos científicos publicados entre 2015 e 2020 (5 anos), sobre o tema da segurança do paciente na antibioticoprofilaxia cirúrgica.

Foram considerados elegíveis os textos aos quais são mencionados, obrigatoriamente, segurança do paciente, antibioticoprofilaxia e centro cirúrgico, sem incluir as especificidades das especialidades cirúrgicas. 
Foram excluídos os protocolos de pesquisa, textos indisponíveis na íntegra, editoriais, teses e dissertações; bem como aqueles estudos que abordavam a temática antibioticoprofilaxia sem estar no contexto cirúrgico.

\section{Seleção e avaliação da qualidade dos estudos}

Realizou-se a triagem inicial baseada nas palavras-chave (primeira etapa). Após a leitura dos títulos e resumos completos, os artigos que não atendiam aos critérios de elegibilidade foram excluídos. Todo o processo de seleção foi realizado de forma independente pelos autores - poucas discordâncias decididas por consenso.

\section{Apresentação dos resultados}

Para a coleta de informações foi elaborado um instrumento contendo os seguintes dados: características (título do artigo, autor, periódico), objetivo do estudo e principais resultados e/ou contribuições. Por último, realizou-se a análise crítica a partir da nuvem de palavras que apresentou a síntese das palavras de maior frequência nos textos que compuseram o corpo da pesquisa, e, depois, classificados em categorias temáticas.

\section{RESULTADOS}

No que tange à pesquisa identificou-se inicialmente 57 referências ( $1^{\mathrm{a}}$ etapa). A partir da leitura completa dos títulos, resumos, e palavras-chave confirmou-se a exclusão de 49 artigos, por não abordarem a temática especifica da segurança do paciente na antibioticoprofilaxia cirúrgicas. Assim, foram excluídos todos os textos que tratavam da antibioticoprofilaxia em especialidades cirúrgicas específica. Não houve nenhum artigo duplicado nas bases de dados Medline e Lilacs (segunda etapa). Ao final, considerando os 
critérios de elegibilidade, restou uma amostra de oito textos, sendo dois textos na base de dados Lilacs e seis na Medline.

Quadro 1. Descrição dos artigos incluídos sobre segurança do paciente na antibioticoprofilaxia cirúrgica

\begin{tabular}{|c|c|c|c|}
\hline Estudo & $\begin{array}{c}\text { Periódico } \\
\text { Ano/Base de } \\
\text { dados }\end{array}$ & Objetivo & Principais resultados \\
\hline $\begin{array}{l}\text { Zhenmi Liu, Jo } \\
\text { C Dumville, Gill } \\
\text { Norman }{ }^{7} \\
\text { Intraoperative } \\
\text { interventions for } \\
\text { preventing } \\
\text { surgical site } \\
\text { infection: an } \\
\text { overview of } \\
\text { Cochrane } \\
\text { Reviews }\end{array}$ & $\begin{array}{l}\text { Cochrane } \\
\text { Database Syst } \\
\text { Rev. 2018. } \\
\text { Pubmed }\end{array}$ & $\begin{array}{l}\text { Apresentar uma visão geral } \\
\text { das revisões Cochrane } \\
\text { sobre a eficácia e } \\
\text { segurança das } \\
\text { intervenções, realizadas } \\
\text { durante o período intra- } \\
\text { operatório, destinadas a } \\
\text { prevenir ISC em todas as } \\
\text { populações submetidas a } \\
\text { cirurgia em uma sala de } \\
\text { operação. }\end{array}$ & $\begin{array}{l}\text { Os antibióticos pré- } \\
\text { operatórios reduzem o } \\
\text { risco de ISC em } \\
\text { comparação com o placebo } \\
\text { após a cirurgia de câncer } \\
\text { de mama. A profilaxia } \\
\text { antibiótica provavelmente } \\
\text { reduz o risco de ISC em } \\
\text { cesarianas em comparação } \\
\text { com a ausência de } \\
\text { antibióticos. A profilaxia } \\
\text { antibiótica provavelmente } \\
\text { reduz o risco de ISC para } \\
\text { correção de hérnia em } \\
\text { comparação com placebo } \\
\text { ou nenhum tratamento. }\end{array}$ \\
\hline $\begin{array}{l}\text { Hilde Valen } \\
\text { Wæhle, Stig } \\
\text { Harthug, Eirik } \\
\text { Søfteland, } \\
\\
\text { Investigation of } \\
\text { perioperative } \\
\text { work processes } \\
\text { in provision of } \\
\text { antibiotic } \\
\text { prophylaxis: a } \\
\text { prospective } \\
\text { descriptive } \\
\text { qualitative study } \\
\text { across surgical } \\
\text { specialties in } \\
\text { Norway }\end{array}$ & $\begin{array}{l}\text { BMJ Open. } \\
2019 . \\
\text { Pubmed }\end{array}$ & $\begin{array}{l}\text { Relatar se o uso da Lista } \\
\text { de Verificação Cirúrgica } \\
\text { Segura (SSC) da OMS } \\
\text { melhora o fornecimento de } \\
\text { SAP e reduz as infecções } \\
\text { no pós-operatório. }\end{array}$ & $\begin{array}{l}\text { O fornecimento do SAP foi } \\
\text { tratado em relação a vários } \\
\text { fatores interativos: } \\
\text { preparação e } \\
\text { administração, precisão da } \\
\text { prescrição, diversidade de } \\
\text { sistemas de pedido de } \\
\text { prescrição, condições } \\
\text { específicas do paciente e } \\
\text { mudanças nos horários da } \\
\text { sala de cirurgia. No } \\
\text { entanto, as verificações de } \\
\text { prescrição foram realizadas } \\
\text { como revisões da Lista de } \\
\text { Verificação Cirúrgica } \\
\text { Segura } \\
\text { SSC formais de itens } \\
\text { profilaxia antibiótica } \\
\text { cirúrgica (SAP) ou como } \\
\text { verificações informais de } \\
\text { documentos relevantes. }\end{array}$ \\
\hline Zenewton A da & Journal of & Avaliar a adesão às boas & A adesão geral foi baixa, \\
\hline
\end{tabular}




\begin{tabular}{|c|c|c|c|}
\hline $\begin{array}{l}\text { Silva Gama, } \\
\text { Pedro J Saturno } \\
\text { Hernandeez, } \\
\text { Marise Reis de } \\
\text { Freitas. } \\
\text { Good infection } \\
\text { prevention } \\
\text { practices in three } \\
\text { Brazilian } \\
\text { hospitals: } \\
\text { Implications for } \\
\text { patient safety } \\
\text { policies }\end{array}$ & $\begin{array}{l}\text { Infection and } \\
\text { Public Health } \\
2019 . \\
\text { Pubmed }\end{array}$ & $\begin{array}{l}\text { práticas de prevenção de } \\
\text { IRAS. }\end{array}$ & $\begin{array}{l}\text { mas maior no hospital } \\
\text { privado, seguido pelas } \\
\text { instituições público- } \\
\text { federais e público- } \\
\text { estaduais. Houve } \\
\text { manutenção adequada dos } \\
\text { cateteres venosos centrais } \\
\text { e alta cobertura vacinal } \\
\text { contra o vírus influenza } \\
\text { entre os profissionais de } \\
\text { saúde. No entanto, a } \\
\text { adesão à higiene das mãos } \\
\text { foi baixa, e a profilaxia } \\
\text { antibiótica cirúrgica e a } \\
\text { prevenção da transmissão } \\
\text { de bactérias } \\
\text { multirresistentes, infecção } \\
\text { do trato urinário por cateter } \\
\text { urinário e associada à } \\
\text { ventilação mecânica foram } \\
\text { inadequadas. }\end{array}$ \\
\hline $\begin{array}{l}\text { Yaser M. } \\
\text { Alahmadi, Raed } \\
\text { H. Alharbi, } \\
\text { Ahmad K. } \\
\text { Aljabri. }^{10} \\
\text { Adherence to the } \\
\text { guidelines for } \\
\text { surgical } \\
\text { antimicrobial } \\
\text { prophylaxis in a } \\
\text { Saudi tertiary } \\
\text { care hospital }\end{array}$ & $\begin{array}{l}\text { J Taibah Univ } \\
\text { Med Sci. } 2020 \\
\text { Pubmed }\end{array}$ & $\begin{array}{l}\text { Avaliar a adesão às } \\
\text { diretrizes para profilaxia } \\
\text { antimicrobiana cirúrgica } \\
\text { em um hospital terciário da } \\
\text { Arábia Saudita. }\end{array}$ & $\begin{array}{l}\text { Cento e trinta e oito } \\
(19,5 \%) \text { pacientes } \\
\text { receberam antibióticos de } \\
\text { acordo com as diretrizes } \\
\text { para profilaxia cirúrgica. } \\
\text { Uma diferença } \\
\text { significativa foi evidente } \\
\text { entre os antibióticos } \\
\text { administrados de acordo } \\
\text { com as diretrizes } \\
\text { recomendadas e a duração } \\
\text { da antibioticoterapia. }\end{array}$ \\
\hline $\begin{array}{l}\text { Mohamedraed } \\
\text { Elshami, Bettina } \\
\text { Bottcher, Issam } \\
\text { Awadallah }{ }^{11} \\
\text { Determinants of } \\
\text { surgeons' } \\
\text { adherence to } \\
\text { preventive } \\
\text { intraoperative } \\
\text { measures of } \\
\text { surgical site }\end{array}$ & $\begin{array}{l}\text { BMC Surg. } \\
2020 . \\
\text { Pubmed }\end{array}$ & $\begin{array}{l}\text { Examinar os fatores que } \\
\text { influenciam a aplicação de } \\
\text { várias medidas preventivas } \\
\text { intraoperatórias de ISC por } \\
\text { cirurgiões e residentes } \\
\text { cirúrgicos na Faixa de } \\
\text { Gaza. }\end{array}$ & $\begin{array}{l}\text { O local e o horário da } \\
\text { operação apresentaram } \\
\text { associações significativas } \\
\text { com a adesão a todas as } \\
\text { medidas preventivas de } \\
\text { ISC, exceto a profilaxia } \\
\text { antibiótica. O tipo de } \\
\text { operação teve uma } \\
\text { associação significativa } \\
\text { com a realização de todas } \\
\text { as medidas, exceto a troca } \\
\text { de instrumentos cirúrgicos. }\end{array}$ \\
\hline
\end{tabular}




\begin{tabular}{|c|c|c|c|}
\hline $\begin{array}{l}\text { infection in } \\
\text { Gaza Strip } \\
\text { hospitals: a } \\
\text { multi-centre } \\
\text { cross-sectional } \\
\text { study } \\
\end{array}$ & & & $\begin{array}{l}\text { A idade do paciente não } \\
\text { teve associação } \\
\text { estatisticamente } \\
\text { significativa com a adesão } \\
\text { a qualquer medida. }\end{array}$ \\
\hline $\begin{array}{l}\text { Massimo } \\
\text { Sartelli, } \\
\text { Leonardo } \\
\text { Pagani, Stefania } \\
\text { Iannazzo }^{12} \\
\text { A proposal for a } \\
\text { comprehensive } \\
\text { approach to } \\
\text { infections across } \\
\text { the surgical } \\
\text { pathway }\end{array}$ & $\begin{array}{l}\text { World J Emerg } \\
\text { Surg. } 2020 \\
\text { Pubmed }\end{array}$ & $\begin{array}{l}\text { Esclarecer questões-chave } \\
\text { na prevenção e tratamento } \\
\text { de infecções em toda a via } \\
\text { cirúrgica, uma força-tarefa } \\
\text { multidisciplinar de } \\
\text { especialistas reuniu-se em } \\
\text { Ancona, Itália, em } 31 \text { de } \\
\text { maio de } 2019 \text {, para um } \\
\text { encontro nacional. Este } \\
\text { documento representa o } \\
\text { resumo executivo das } \\
\text { declarações finais } \\
\text { aprovadas pelo painel de } \\
\text { especialistas. }\end{array}$ & $\begin{array}{l}\text { Um acordo sobre as } \\
\text { declarações foi alcançado } \\
\text { pelo método Delphi. As } \\
\text { declarações foram } \\
\text { aprovadas com } \\
\text { concordância } \geq \\
80 \% \text {. Depois da reunião, o } \\
\text { painel de especialistas se } \\
\text { reuniu por e-mail para } \\
\text { preparar e revisar o } \\
\text { documento de consenso } \\
\text { resultante da reunião. O } \\
\text { manuscrito foi revisado } \\
\text { sucessivamente por todos } \\
\text { os membros e finalmente } \\
\text { revisado como o presente } \\
\text { manuscrito. Este } \\
\text { documento representa o } \\
\text { resumo executivo das } \\
\text { declarações finais } \\
\text { aprovadas pelo painel de } \\
\text { especialistas. }\end{array}$ \\
\hline $\begin{array}{l}\text { Matheus Santos } \\
\text { Melo, Thialla } \\
\text { Andrade } \\
\text { Carvalho, Maria } \\
\text { Cláudia Tavares } \\
\text { Mattos }{ }^{13} \\
\text { Evaluation of } \\
\text { surgical } \\
\text { antimicrobial } \\
\text { prophylaxis in a } \\
\text { teaching hospital }\end{array}$ & $\begin{array}{l}\text { Journal of } \\
\text { Epidemiology } \\
\text { and Infection } \\
\text { Control. } 2019 . \\
\text { Lilacs }\end{array}$ & $\begin{array}{l}\text { Avaliar } \\
\text { profilaxia antimicrobiana } \\
\text { no perioperatório de } \\
\text { cirurgias eletivas } \\
\text { realizadas em um hospital } \\
\text { de ensino de Sergipe. }\end{array}$ & $\begin{array}{l}\text { A profilaxia antimicrobian } \\
\text { a foi utilizada em } 78,80 \% \\
\text { das cirurgias, seguiu } \\
\text { à padronização do serviço } \\
\text { de controle de } \\
\text { infecção quanto a seleção } \\
\text { do antibiótico }(91,80 \%) \text {, } \\
\text { administrada no momento } \\
\text { ideal (10,90\%) e } \\
\text { considerada adequada } \\
\text { (escolha do antibiótico, } \\
\text { momento } \\
\text { da administração e duração } \\
\text { da profilaxia) (4,40\%). A } \\
\text { classe de antibiótico de } \\
\text { maior escolha foi a } \\
\text { das cefalosporinas (93,60 } \\
\text { \%) e o repique foi } \\
\text { realizado em somente } \\
\text { 22,20\% das cirurgias que } \\
\text { tinham indicação. }\end{array}$ \\
\hline Fernanda do & Revista & Identificar a prática & Constatou-se que em 81 \\
\hline
\end{tabular}




\begin{tabular}{|c|c|c|c|}
\hline $\begin{array}{l}\text { Prado Tostes, } \\
\text { Maria; Maran, } \\
\text { Edilaine; Sorrilh } \\
\text { a Raimundo, } \\
\text { Larissa }{ }^{14} \\
\text { Prática da } \\
\text { profilaxia } \\
\text { antimicrobiana } \\
\text { cirúrgica como } \\
\text { fator de } \\
\text { segurança do } \\
\text { paciente }\end{array}$ & $\begin{array}{l}\text { SOBECC } 2016 \\
\text { Lilacs }\end{array}$ & $\begin{array}{l}\text { da profilaxia antimicrobian } \\
\text { a cirúrgica adotada } \\
\text { pelos profissionais atuante } \\
\text { s em centro cirúrgico. }\end{array}$ & $\begin{array}{l}\text { (81\%) das cirurgias, } \\
\text { limpas, potencialmente } \\
\text { contaminadas e } \\
\text { contaminadas, } \\
\text { a profilaxia antimicrobiana } \\
\text { cirúrgica foi realizada. } \\
\text { Entretanto, na maioria } \\
\text { delas (54/66,6\%), o } \\
\text { antimicrobiano não foi } \\
\text { administrado dentro de } \\
\text { uma hora antes da incisão } \\
\text { cirúrgica. Adicionalmente, } \\
\text { em seis (18,1\%) cirurgias } \\
\text { potencialmente } \\
\text { contaminadas e em uma } \\
\text { (33\%) contaminada, em } \\
\text { que seu uso é } \\
\text { indispensável, o } \\
\text { antimicrobiano não foi } \\
\text { utilizado. }\end{array}$ \\
\hline
\end{tabular}

A abordagem dos 8 artigos selecionados para compor as duas categorias temáticas deste estudo, foram transferidas para um documento txt traduzidos para o português. Em seguida, o arquivo txt foi enviado ao site gerador de nuvem de palavras, formando a nuvem abaixo. Foram retiradas manualmente expressões, conectivos, e expressões que apenas tinham intuito de dar coesão ao texto.

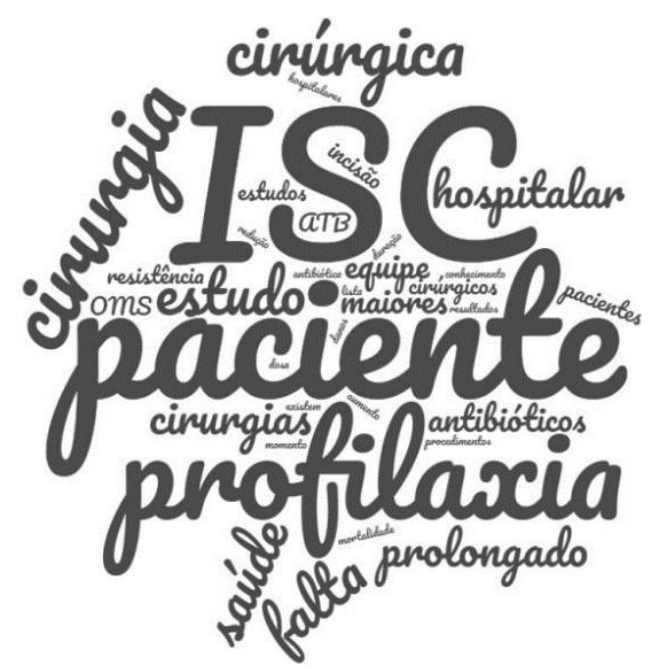

Imagem 2. Nuvem de palavras a partir da análise de artigos 


\section{DISCUSSÃO}

\section{Antibioticoprofilaxia e infecção de sítio cirúrgico}

A ISC (infecção de sítio cirúrgico) é um dano muito sério que leva inúmeras morbidades anualmente, sendo que em muitos casos é necessário uma reabordagem cirúrgica, outros um tempo prolongado de antibióticos, uma hospitalização mais demorada, podendo ainda levar a morte. De acordo com dados apresentados pelo Instituto Nacional de Excelência em Saúde e Cuidados do Reino Unido (NICE), alguns estudos realizados no Reino Unido apontam que a incidência de ISC aumentam em até 10 dias a hospitalização de um paciente, gerando maiores custos a saúde. Outra pesquisa realizada nos Estados Unidos apontou que, os aumentos dos gastos com esses pacientes em um período de um ano chegaram a custar mais de U\$ $\$ 1,6$ bilhões ${ }^{7}$.

As maiores taxas de ISC estão em países de baixa e média renda, nos EUA estão associadas como as maiores causas de infecções mais comuns, e em segunda posição está a Europa. Já está bem estruturada e aceita a questão da profilaxia antibiótica segura (SAP), e é vista como uma das maneiras mais eficientes na prevenção para ISC, de acordo com a diretrizes da Organização Mundial da Saúde (OMS). A depender do tipo de cirurgia (limpa, contaminada, infectada) tempo de cirurgia, e as condições do paciente, existe a administração do antibiótico de acordo com o que o paciente pode receber, sendo que em alguns casos é preciso sua administração ser repetida no intra operatório (nos casos de cirurgia muito longa) pois é preciso que o antibiótico alcance suas propriedades de concentrações respeitando tempo e intervalo baseado na farmacocinética de cada antibiótico ${ }^{8}$.

Após a cirurgia ainda é considerado uma profilaxia, podendo essa ser estendida até 48 horas após o procedimento cirúrgico. A dose, horário de sua administração, e se haverá repetição no ato cirúrgico, estão associados a fatores relacionados ao paciente e a escolha do 
médico prescritor. Uma administração tardia, ou antes do momento adequado, causam aos pacientes danos, os quais poderiam ser evitados. O prolongamento de SAP não são recomendações das diretrizes para prevenir ISC, pois pode gerar ao paciente uma resistência antimicrobiana, e isso vem sendo realizado por mais de 24 horas, em muitos centros de saúde ${ }^{8}$.

A ISC é dita como o tipo de infecção mais comum, de ser encontrada em ambientes hospitalares. Os graves resultados obtidos devido a essas infecções fazem com que exista a necessidade de empenhar esforços, na luta para prevenção de tais no pós-operatório. Independente da prescrição, a porcentagem de administração de SAP está em 96,7\%, basicamente o maior intuito dessa administração é prevenir infecções e complicações pósoperatórias ${ }^{9,10}$. Alguns estudos mostram que as ISC estão relacionadas a fatores do próprio paciente como, idade avançada, sendo que existe outra linha de estudos que defende que isso não seja um fator que tenha um impacto significativo para ISC. Mesmo que não seja um fator tão expressivo, este pode se relacionar com outros fatores associados ao procedimento. ${ }^{11}$

A prevenção de ISC consiste em um trabalho em equipe, a colaboração de cada profissional acrescenta de forma a empenhar e praticar as melhores práticas através de seus conhecimentos ao paciente, em busca da segurança do paciente. Existe um empenho em todo o mundo para melhorar essa segurança, no que aborda ISC e resistência antimicrobiana, sendo que pacientes submetidos a procedimentos cirúrgicos estão suscetíveis a adquirirem ISC, por isso existem as diretrizes e protocolos que tentam combater números crescentes de infecções ${ }^{12}$.

Uma das principais infecções encontradas no Brasil, sendo responsável por 14\% a $16 \%$ das infecções relacionadas as internações, a ISC é considerada um evento adverso cirúrgico grave. Medidas adotadas para prevenir essas infecções, como a administração de antibiótico como profilaxia, é indispensável para vários procedimentos cirúrgicos, consiste na 
administração endovenosa de determinado antibiótico de forma profilática, com intuito de que atinja níveis de concentração adequados durante a incisão cirúrgica, visando uma redução de carga microbiana na contaminação intraoperatoria, para que alcance o seu ideal, é preconizada sua administração em até 60 minutos antes da incisão cirúrgica ${ }^{13}$.

\section{Lista de Verificação Segura}

Foi desenvolvida a lista de verificação cirúrgica (SSC), pela OMS, e esta é aplicada e estabelecida como critério para realização de cirurgias no intuito de fornecer segurança ao paciente. Essa lista inclui critérios baseados em evidências eficazes para combater infecções cirúrgicas, já existem relatos que a introdução da lista nas salas cirúrgicas diminui os riscos de morbidade, complicações e até mesmo mortalidade, reduzindo as ISC. Um estudo comprova que, a implantação de alguns cuidados peri operatórios, como implantação da SSC da OMS teve melhoria significativa na administração adequada de $\mathrm{SAP}^{8}$.

A existência de diretrizes e normas, são basicamente para proporcionar uma segurança ao paciente e também equipe, infelizmente alguns resultados apontam a não adesão de alguns profissionais no que tange a SAP.A realização de SAP 60 minutos antes da incisão cirúrgica tem por objetivo prevenir a ISC, através do fornecimento adequado da quantidade suficiente para os tecidos antes da incisão, o respeito do tempo é algo essencial. De acordo com a Sociedade Americana de Farmacêuticos do Sistema de Saúde (ASHP), foi divulgado diretrizes terapêuticas relacionadas a seleção, momento e duração do SAP no intuito de contribuir com a redução de morbidade e mortalidade relacionados a ISC $^{10}$.

A SAP está como uma das recomendações da OMS para cirurgia segura, no entanto alguns estudos apontam para a não aceitação da profilaxia. Um estudo brasileiro aponta uma adesão maior em relação a estudos italianos. O êxito da profilaxia depende do tipo de cirurgia, visto isso o cirurgião saberá se prescreve ou não e qual a melhor escolha para o procedimento 
a ser realizado no combate a ISC. Foi registrada uma porcentagem de $18 \%$ relacionada ao uso inadequado de antibiótico profilático em cirurgias. Há ainda, um déficit de conhecimento por parte dos cirurgiões relacionada a SAP, muitos por falta de observarem as diretrizes relacionadas a prevenção de ISC. Assim como existe a falta de aplicabilidade de profilaxia, há também o excesso de aplicabilidade (tempo prolongado) ${ }^{11}$.

$\mathrm{O}$ aumento nos números associados a ISC tem crescido de forma preocupante, sendo responsáveis por maiores tempos de internação, exigindo intervenções médicas somado a maiores custos hospitalares resultando em morbidades e mortalidades. As diretrizes publicadas pela OMS, para prevenção de ISC incluem 13 recomendações voltadas ao período pré-operatório, e 16 para prevenção durante e após a cirurgia. Essas diretrizes consistem desde o básico, como o banho do paciente antes da cirurgia, como lavagem das mãos das equipes cirúrgicas, antibioticoprofilaxia, até o tipo de sutura adequado ${ }^{12}$.

A implantação da lista de verificação cirúrgica (SSC) é um processo que envolve ações de uma equipe multidisciplinar, em todos os tempos (antes, durante e após a cirurgia), funciona de forma sincronizada, onde cada profissional age no seu tempo, realizando as atividades que necessitam, sendo que a SAP está inclusa nessa lista e deve ser observada por $\operatorname{todos}^{8}$

De acordo com Liu, a aplicação da antibioticoprofilaxia endovenosa em pacientes que serão submetidos a alguma intervenção cirúrgica são eficazes na prevenção de ISC. Em um estudo realizado num ensaio com 99 participantes, onde foi aplicado a profilaxia antibiótica e outro grupo com nenhuma profilaxia, foi observado que o grupo que recebeu a profilaxia houve redução nos índices de ISC. No que tange a mortalidade ou qualidade de vida, com base nesse estudo aplicado em Cochrane não se pode afirmar se existe um efeito negativo, é necessário a aplicação desse ensaio mais afundo e um acompanhamento mais prolongado ${ }^{7}$. 
Segundo estudos, existe uma adesão de $71 \%$ da administração de antibioticoprofilaxia cirúrgica, mesmo em centros cirúrgicos onde não exista essa diretriz consolidada, as maiores falhas estão relacionadas a indicação e tempo inadequada. Um estudo realizado em um hospital no Centro Oeste brasileiro, identificou 25\% de prescrições em conformidade com a necessidade, dentre essa porcentagem perceberam $100 \%$ de adesão ao momento apropriado para administrar a profilaxia segura ${ }^{9}$.

Existem danos que estão relacionados a administração inadequada, como, grande incidência da resistência antimicrobiana em pacientes pós cirúrgicos, devido ao tempo prorrogado de internação hospitalar, gerando maiores custos ao paciente (quando privado), e custos elevados ao sistema de saúde. Dentre as diretrizes relacionadas a administração de SAP, a ASHP observa que o momento adequado para SAP é dentre os 60 minutos que antecedem a incisão cirúrgica, e a escolha de quantidade a ser administrada deve ser calculada e escolhida de acordo com peso, e as condições do paciente, assim como a sua repetição para procedimentos com maior duração. Por recomendações da Sociedade Americana, cabem também o uso reduzido de antibiótico no pós-operatório, por menos de 24 horas, ou em única dose. Tanto procedimento cirúrgico em adultos como em crianças existem sim a necessidade de realização de SAP, conforme necessidade de cada um, e para minimizar morbidade e mortalidade relacionados a ISC, diminuindo também os custos com a saúde, dentre elas tempo de internação ${ }^{10}$.

\section{Tempo de administração de antibiótico}

Alguns indícios apontam que $60 \%$ das infecções poderiam ser evitáveis, sendo que uma das formas mais comuns de profilaxia é o uso de antibióticos, de forma racional e no tempo certo. Por parecer ser algo de simples administração, muitas vezes, ou na maioria das vezes, ela não atinge sua eficácia, pela administração fora do tempo, uso inadequado, ou 
tempo prolongado no pós operatório, aumentado então a incidência de ISC, e causando danos ao paciente, como tempo prolongado do uso de antibiótico, morbidades, maiores custos hospitalares, e o óbito ${ }^{13}$.

A administração de SAP dentro do tempo estabelecido é eficaz, um estudo realizado no Canadá demonstra que o fato de ser uma país mais desenvolvido e com economia mais elevada que o Brasil apresenta taxas de 5,9\% de adesão ao tempo apropriado na administração, semelhante ao Brasil que possui uma variabilidade alta relacionada ao tempo de administração, e essa variabilidade no tempo (acima de 2 horas) acarreta consequências. Um estudo que relacionava ISC ao momento correto de profilaxia, mostrou que um tempo maior da administração do antibiótico (acima de duas horas) e a incisão da pele aumentou em 6,7 vezes os índices de ISC $^{14}$.

A importância da administração administrada 60 minutos antes da incisão cirúrgica é muito importante, porém vale ressaltar sobre a suspensão no pós-operatório destes antibióticos, dentro de um prazo de até 24 horas em grande parte dos procedimentos, e especificamente nas cirúrgicas de até 48 horas. Em relação as cirurgias de grande duração, essas superiores a 3 horas existe a recomendação de uma repetição de dose durante o procedimento. Alguns estudos apontam que dose única de administração de SAP e uso prolongado de 24 horas, foi notada a mesma eficácia em abas as abordagens. Uma profilaxia com tempo ampliado não é tão vantajosa, pois acarreta alguns problemas como resistência antimicrobiana, altos custos hospitalares e maior tempo de internação ${ }^{10}$.

\section{Uso prolongado de antibiótico, devido despreparo/falha da equipe.}

A segurança do paciente consiste em um cuidado continuado, lembrando que o cuidado, o momento, a pessoa e a dose certa contribuem diretamente para resultados positivos, que consistem em uma assistência da equipe multidisciplinar baseada em 
evidências científicas bem fundamentadas. As práticas relacionadas a administração de profilaxia segura, estão pautadas em diretrizes pela OMS que lançou o Desafio Global para Segurança do Paciente, tendo como tema alvo a segurança do paciente, que atua na prevenção de ISC. Constam itens que estão agrupados em um documento chamado de checklist, ou lista de verificação de cirurgia segura (SSC), onde constam etapas a serem cumpridas antes, durante e após a cirurgia, para promover ao paciente e aos profissionais de saúde uma segurança maior. De acordo com Tostes, a adesão a SAP, está muito abaixo do ideal, sendo uma evidência comprovada como uma das medidas preventivas mais adequadas no combate a ISC, sendo essas infecções um problema pontual, e evitável ${ }^{14}$.

Como a profilaxia antimicrobiana é uma das medidas indispensáveis na prevenção das ISC, a não administração nos casos que possuem indicação é inaceitável e pode contribuir para o aumento da incidência das infecções relacionadas à assistência à saúde comprometendo a qualidade da assistência e a segurança do paciente. Do mesmo modo, a administração indiscriminada de profilaxia antimicrobiana na ausência de indicação, contribui para uma exposição do paciente a uma possível resistência antimicrobiana ${ }^{13}$.

A falta de conhecimento relacionado as diretrizes, que servem como um guia para segurança do paciente, é um fator muito complexo, um estudo realizado em países em desenvolvimento constatou o uso bem reduzido e aplicação de SAP, sendo que apenas $51 \%$ dos residentes em cirurgia tem conhecimento associados as diretrizes ${ }^{10}$.

Não são todos os procedimentos cirúrgicos que necessitam de antibioticoprofilaxia, os procedimentos classificados como limpos por exemplo (onde não haja implantação de prótese ou não existam comorbidades relacionadas ao paciente), não é necessário a administração da profilaxia antibiótica, porém um estudo realizado no Hospital de Ensino de Sergipe, apontou que o uso de profilaxia está indo contra as diretrizes recomendadas pela OMS, aplicando de forma não necessária. Já a omissão na administração de antibióticos é uma medida 
inaceitável, o que pode aumentar a incidência de ISC, comprometendo a segurança do paciente. O certo é que, os casos não indicados pelas diretrizes, devem ser observados, assim como o prolongamento desnecessário de administração, pois é através deste prolongamento que há a resistência antimicrobiana ${ }^{13}$.

Visto as falhas relacionadas a SAP dentro dos centros cirúrgicos, é necessário um envolvimento institucional, com avaliações e feedbacks aos profissionais que aplicam o checklist, e administram o antibiótico para observarem e perceberem tamanha importância do cumprimento de tal diretriz. Na equipe de enfermagem é visto uma preocupação maior, e uma observância mais alta que a comparada as outras especialidades. Alguns médicos são relutantes na implantação de protocolos, pois para muitos deles seus conhecimentos e especialidades valem mais, sendo que não é assim, protocolos baseados em evidencias são totalmente eficazes para prática segura ${ }^{14}$.

É essencial que os centros cirúrgicos, e todo o ambiente hospitalar, faça adesão a implantar projetos referentes a uso de antibiótico, abrangendo a importância na redução do uso de ATB, adesão a profilaxia antibiótica, aplicação das diretrizes sobre ATB, e o uso inapropriado de ATB, não apenas em algumas esferas, mas em âmbito nacional ${ }^{10}$.

Pela falta de conhecimento, muitos aderem a profilaxia antibiótica pós cirúrgica de forma prolongada, mostrando uma não conformidade entre as diretrizes e a prática aplicada, não apenas a falta de conhecimento como também falta de recursos, discordância entre a equipe médica devido à ausência de conhecimento sobre determinado assunto, assim como informações que estão ultrapassadas e que as diretrizes provam que não é o correto para o paciente sendo ainda uma prática comum, como exemplo temos, o uso prolongado de antibiótico para evitar ISC. Uma pesquisa realizada em um hospital terciário em Almadinah Almunawwarah, no oeste do KSA (Arábia Saudita), sobre adesão e administração de SAP, os resultados mostram que não estavam em conformidade com as diretrizes recomendadas pela 
ASHP, sendo que os participantes receberam profilaxia no pré e/ou pós operatório. De acordo com os resultados foi visto que 19,5\% não estavam de acordo com as diretrizes, onde apenas 22,5\% do SAP foi administrado dentro do prazo estipulado. Já outro estudo realizado em KSA, na capital, foi observado um respeito total à administração correta relacionada ao tempo. A não observância relacionada à espera do tempo de administração de antibiótico, revela o não respeito as práticas recomendadas na diretriz de ASHP, indicando maiores riscos relacionados a infecções, em ambientes de saúde ${ }^{10}$.

A falta de treinamento em equipe, capacitação profissional para implementação das diretrizes é sem dúvidas um fator que implica significativamente nos resultados a prevenção a ISC. Ainda é controverso o uso de SAP na prevenção de ISC. Quando existe um tempo muito longo de cirurgia, não apenas pelo procedimento, mas relacionado ao desgaste da equipe, em ênfase o cirurgião, pode levar a uma exaustão gerando assim a falta de observar alguns detalhes que são primordiais na prevenção de ISC. Os cirurgiões são um dos fatores de extrema importância na redução de taxas relacionadas a ISC, aprimorar conhecimentos através de educação continuada com ênfase em medidas que podem ser adotadas na prevenção de ISC, pode interferir na qualidade do serviço prestado, gerando uma segurança maior ao paciente ${ }^{11}$.

Há uma falta de aceitação dos profissionais de saúde relacionado a SAP. A falta de protocolos nas instituições de saúde que tem abrangência sobre a temática, o costume que alguns carregam de que apenas antibióticos de longa duração e de nível alto são mais eficientes que os de curta duração, o uso prolongado de ATB(antibiótico), assim como o costume de determinados tipos, pois estes estão a mais tempo no ambiente hospitalar, são pontos que contribuem para adesão inadequada das diretrizes relacionadas a SAP. Em vista disto, a prescrição, reduzir o uso inadequado de ATB, como implementar as diretrizes pertinentes a temática é benéfica ao paciente tanto na profilaxia como terapia, impactando 
diretamente a segurança do paciente. Existe de fato uma divergência em relação as diretrizes e o que é realizado no ambiente hospitalar, referente ao uso de ATB, é imprescindível diretrizes baseadas em evidencias para implantar a administração de SAP nos hospitais, baseado nas resistências antimicrobianas, visando a segurança do paciente ${ }^{10}$.

É essencial que os centros cirúrgicos, e todo o ambiente hospitalar, faça adesão a implantar projetos referentes a uso de antibiótico, abrangendo a importância na redução do uso de ATB, adesão a profilaxia antibiótica, aplicação das diretrizes sobre ATB, e o uso inapropriado de ATB, não apenas em algumas esferas, mas em âmbito mundial, observadas as diretrizes divulgadas pela OMS para cirurgia segura ${ }^{10}$.

\section{CONCLUSÃO}

Com base nas publicações, conclui-se que os cuidados relacionados à segurança do paciente na antibioticoprofilaxia nas cirurgias, estão diretamente relacionados ao cumprimento das diretrizes publicado pela OMS, e estes implicam na redução dos casos relacionados a infecção de sítio cirúrgico, tal como diminuição das taxas de complicações e mortalidade.

Em virtude disto, a lista de verificação segura cumpre o papel de uma maior segurança nos cuidados mediatos ao momento cirúrgico, bem como o tempo de administração no prazo de até 60 minutos antes da incisão cirúrgica atinge a concentração adequada para eficácia antibiótica, e que o uso prolongado de antibiótico não favorece ao paciente, causando danos, tais como resistência antimicrobiana, devido ao despreparo da equipe, ou falhas na sua prescrição. 


\section{REFERÊNCIAS}

1. Organização Mundial da Saúde. Manual de implementação para apoiar a prevenção de infecções de sítio cirúrgico no nível de instalação - transformando as recomendações em prática. Geneva. 2018.

2. Oliveira AC, Silva MVG. Teoria e prática na prevenção da infecção do sítio cirúrgico. $1^{\circ}$ Edição. São Paulo: Editora Manole LTDA. 2015.

3. BRASIL. Agência Nacional de Vigilância Sanitária. Diretriz Nacional para Elaboração de Programa de Gerenciamento do uso de antimicrobianos em Serviços de Saúde. 2017.

4. Barlam TF, Cosgrove SE, Abbo LM; Macdougall C, Schuetz AN, Septimus EJ, et al. Implementing an Antibiotic Stewardship Program: Guidelines by the Infectious Diseases Society of America and the Society for Healthcare Epidemiology of America. Clinical Infectious Diseases. [periódicos na Internet] 2016. Disponível em: https://academic.oup.com/cid/article/62/10/e51/2462846.

5. Macedo TR, Rocha PK, Tomazoni A, Souza S, Anders JC, Davis K. The culture of patient safety from the perspective of the pediatric emergency nursing team. Rev Esc Enferm USP. [periódicos na Internet] 2016;50(5):756-762. DOI: http://dx.doi.org/10.1590/S0080$\underline{623420160000600007 .}$

6. Galvão TF, Pansani TSA, Harrad D. Principais itens para relatar revisões sistemáticas e metaanálises: a recomendação prisma. Epidemiol Serv Saúde. [periódicos na Internet] 2015. 24(2):335-42. DOI: 10.5123/s1679-49742015000200017. Disponível em: https://www.scielo.br/scielo.php?script=sci_arttext\&pid=S2237-96222015000200335. Acesso em: 13 agosto 2019.

7. Liu Z, Dumville JC, Norman G, Westby MJ, Blazeby J, McFarlane E, et al. Intraoperative interventions for preventing surgical site infection: an overview of Cochrane Reviews. 
Cochrane Database Syst Rev. [periódicos na Internet] 2018. [acesso em 3 de Set 2020]; Disponível em: https://www.ncbi.nlm.nih.gov/pmc/articles/PMC6491077/.

8. Waehle HV, Harthug S, Softeland E, Sevdalis N, Smith I, Wiig S, et al. Investigation of perioperative work processes in provision of antibiotic prophylaxis: a prospective descriptive qualitative study across surgical specialties in Norway. BMJ Open. [periódicos na Internet] 2019. [acesso em 4 Set 2020] Disponível em: https://www.ncbi.nlm.nih.gov/pmc/articles/PMC6596935/.

9. Gama ZAS, Hernandez PJS, Freitas MR., Padoveze MC, Saraiva COPO, Paulino LG, et al. Good infection prevention practices in three Brazilian hospitals: Implications for patient safety policies. J Infect Public Health. [Periódicos na Internet] 2019. [Acesso em 5 Set 2020] Disponível em: https://www.sciencedirect.com/science/article/pii/S1876034119300875?via\%3Dihub.

10. Alahmadi YM, Alharbi RH, Aljabri AK, Alofi FS, Alshaalani OA, Alssdi BH. Adherence to the guidelines for surgical antimicrobial prophylaxis in a Saudi tertiary care hospital. J Taibah Univ Med Sci. [Periódicos na Internet] 2020 [ acesso em 5 Set de 2020] 2020 Mar 5;15(2):136-141. Disponível em: https://www.ncbi.nlm.nih.gov/pmc/articles/PMC7184216/ .

11. Elshami M, Bottcher B, Awadallah I, Alnaji A, Aljedaili B, Abu Sulttan H, Hwaihi M. Determinants of surgeons' adherence to preventive intraoperative measures of surgical site infection in Gaza Strip hospitals: a multi-centre cross-sectional study. BMC Surg. [Periódicos na Internet] 2020 [ acesso em 7 Set de 2020] Jan 30;20(1):21. doi: 10.1186/s12893-020-06844. Disponível em: https://www.ncbi.nlm.nih.gov/pmc/articles/PMC6993375/ .

12. Sartelli M, Pagani L, Catena F, Moro ML, Viale P, Pan A. et al. A proposal for a comprehensive approach to infections across the surgical pathway. World J Emerg Surg. [Periódicos na Internet] 2020. [ acesso em 7 Set de 2020]; Disponível em: https://wjes.biomedcentral.com/articles/10.1186/s13017-020-00295-3 . 
13. Melo MS, Carvalho TA, Mattos MCT, Campos MPA, Mendonça SB, Lobo IMF. Avaliação da profilaxia antimicrobiana cirúrgica em um hospital de ensino. Journal of Epidemiology and Infection Control. [Periódicos na Internet] 2019. [ acesso em 7 Set de 2020]. Santa Cruz do Sul, 2019 Jan-Mar;9(1):75-80.

Disponível em: https://online.unisc.br/seer/index.php/epidemiologia/article/view/11461 .

14. Tostes M, Maran E, Raimundo L, Mai L. Prática da profilaxia antimicrobiana cirúrgica como fator de segurança do paciente. Revista SOBECC [Internet]. 2016 Jun 8; [Citado em 2021 Out 26]; 21(1): 13-21. Disponível em: https://revista.sobecc.org.br/sobecc/article/view/37. 